\title{
Hadron-quark Phase Transition in Hybrid Stars with the Field Correlator Method
}

\author{
G. F. Burgio* \\ INFN Sezione di Catania. Via S. Sofia 64, I-95123 Catania, Italy \\ E-mail: Eiorella.burgiodct.infn.it \\ D. Zappalà \\ INFN Sezione di Catania, Via S. Sofia 64, I-95123 Catania, Italy \\ E-mail: dario.zappaladct.infn.it
}

\begin{abstract}
We explore the structure of hybrid stars based on a quark matter equation of state (EoS) built with the Field Correlator Method (FCM). For the hadronic phase, we use the microscopic BruecknerHartree-Fock (BHF) many-body theory, and its relativistic counterpart, i.e. the Dirac-Brueckner (DBHF). We find that the main features of the phase transition are directly related to the FCM parameters, i.e. the quark-antiquark potential $V_{1}$, the gluon condensate $G_{2}$ and the color-flavour superconducting gap $\Delta$, and that the FCM EoS can be accurately represented by the CSS (constant speed of sound) parametrization. We display the mapping between the FCM EoS and the CSS scheme, and demonstrate that it holds true even in the case of paired quark matter.
\end{abstract}

The Modern Physics of Compact Stars 2015

30 September 2015 - 3 October 2015

Yerevan, Armenia

\footnotetext{
*Speaker.
} 


\section{Introduction}

The appearance of quark matter in the interior of massive neutron stars (NS) is one of the mostly debated issues in the physics of these compact objects. Many equations of state (EoS) have been used to describe the interior of NS. If we consider only purely nucleonic degrees of freedom and the EoS is derived within microscopic approaches [四, it turns out that for the heaviest NS, close to the maximum mass (about two solar masses), the central particle density reaches values larger than $1 / \mathrm{fm}^{3}$. In this density range the nucleon cores (dimension $\approx 0.5 \mathrm{fm}$ ) start to touch each other, losing their identity, and quark degrees of freedom are excited at a macroscopic level. Unfortunately, while the microscopic theory of the nucleonic EoS has reached a high degree of sophistication [ [2], 目, 团, [], 目], the quark matter (QM) EoS is still poorly known at zero temperature and at the high baryonic density appropriate for NS. In fact the essential theoretical tool, i.e. lattice formulation of the quantum chromodynamics (QCD) is inapplicable at large baryon densities and small temperature due to the so-called Sign Problem [ $\square]$, and this is due to its complicated nonlinear and nonperturbative nature. On the other hand, in the large temperature and small density region lattice QCD simulations have provided controlled results for the EoS as well as for the nature of the transition $[\mathbb{8}, \mathbb{Q}]$.

Unfortunately it is not straightforward to predict the relevance of quark degrees of freedom in the interior of NS for the various physical observables, like cooling evolution, glitch characteristics, neutrino emissivity, and so on. The value of the maximum mass of NS is probably one of the physical quantities that is most sensitive to the presence of quark matter in NS. The recent observation of a large NS mass in PSR J0348+0432 with mass $\mathrm{M}=2.01 \pm 0.04 \mathrm{M}_{\odot}\left(M_{\odot}=2 \times 10^{33} \mathrm{~g}\right)$ [ए]] implies that the EoS of NS matter is stiff enough to keep the maximum mass at these large values. Purely nucleonic EoS are able to accommodate such large masses [W]. Since the presence of non-nucleonic degrees of freedom, like hyperons and quarks, tends usually to soften considerably the EoS with respect to purely nucleonic matter, thus lowering the mass value, their appearance would in this case be incompatible with observations. The large value of the mass could then be explained only if both hyperonic and quark matter EoS are stiffer than expected.

Many models of quark matter do exist, and they all contain a high degree of uncertainty. The best one can do is to compare the predictions of different models and to estimate the uncertainty of the results for the NS matter as well as for the NS structure and mass. In this paper we use the Field Correlator Model (FCM) for the quark EoS [ए]], which in principle is able to cover the full temperature-chemical potential plane. The FCM EoS contains ab initio the property of confinement, which is expected to play a role as far as the stability of a neutron star is concerned [ए2]. This model can be also tested against NS observations [[13], which could seriously constrain the model parameters, i.e. the quark-antiquark potential $V_{1}$ and the gluon condensate $G_{2}$. Recently, we found that the FCM model can be expressed in the language of the "Constant Speed of Sound" (CSS) parametrization [44, [5]], and we showed how its parameters can be mapped on to the CSS parameter space. We remind that the CSS scheme is a general parametrization suitable for expressing experimental constraints in a model-independent way, and for classifying different models of quark matter and establishing connections among them. It is applicable to high-density equations of state for which: (a) there is a sharp interface between nuclear matter and quark matter, (b) the speed of sound in the high-density matter is pressure-independent in the range between the first- 
order transition pressure up to the maximum central pressure of neutron stars. Given the nuclear matter $\operatorname{EoS} \varepsilon_{\mathrm{NM}}(p)$, the high-density EoS can be expressed as

$$
\varepsilon(p)= \begin{cases}\varepsilon_{\mathrm{NM}}(p) & p<p_{\text {trans }} \\ \varepsilon_{\mathrm{NM}}\left(p_{\text {trans }}\right)+\Delta \varepsilon+c_{\mathrm{QM}}^{-2}\left(p-p_{\text {trans }}\right) & p>p_{\text {trans }}\end{cases}
$$

where the three parameters: the pressure $p_{\text {trans }}$ at the transition, the discontinuity in energy density $\Delta \varepsilon$ at the transition, and the speed of sound $c_{Q M}$ characterize completely the high-density phase.

As far as the hadronic phase is concerning, we use two definite EoS, based on the BruecknerHartree-Fock many-body theory for nuclear matter, both in its non-relativistic and relativistic version. Those are briefly reviewed in the next Section. In Sect. 3 we illustrate the FCM at finite temperature and density, with the inclusion of the color-flavour locking effect. Sect. 4 contains numerical results, with some general results on the hadron-quark phase transition as described by the CSS parametrization. In Sect.5 we discuss the FCM mapping onto the CSS parametrization and the consequent mass-radius-central density relation for hybrid stars. Effects due to the inclusion of hyperons are also briefly mentioned. Conclusions are reported in Sect.6.

\section{Hadronic Phase: EoS in the Brueckner-Bethe-Goldstone theory}

The BHF method for the nuclear matter EoS is based on the Brueckner-Bethe-Goldstone (BBG) many-body theory, which is the linked cluster expansion of the energy per nucleon of nuclear matter (see Ref.[ए]], chapter 1 and references therein). In this approach one systematically replaces the bare nucleon-nucleon $(\mathrm{NN})$ interaction $V$ by the Brueckner reaction matrix $G$, which is the solution of the Bethe-Goldstone equation

$$
G(\rho ; \omega)=V+V \sum_{k_{a} k_{b}} \frac{\left|k_{a} k_{b}\right\rangle Q\left\langle k_{a} k_{b}\right|}{\omega-e\left(k_{a}\right)-e\left(k_{b}\right)} G(\rho ; \omega)
$$

where $\rho$ is the nucleon number density, $\omega$ is the starting energy, and $\left|k_{a} k_{b}\right\rangle Q\left\langle k_{a} k_{b}\right|$ is the Pauli operator. $e(k)=e(k ; \rho)=\frac{\hbar^{2} k^{2}}{2 m}+U(k ; \rho)$ is the single particle energy, and $U$ is the single-particle potential,

$$
U(k ; \rho)=\sum_{k^{\prime} \leq k_{F}}\left\langle k k^{\prime}\left|G\left(\rho ; e(k)+e\left(k^{\prime}\right)\right)\right| k k^{\prime}\right\rangle_{a}
$$

The subscript " $a$ " indicates antisymmetrization of the matrix element. In the BHF approximation the energy per nucleon is

$$
\frac{E}{A}(\rho)=\frac{3}{5} \frac{\hbar^{2} k_{F}^{2}}{2 m}+\frac{1}{2 A} \sum_{k, k^{\prime} \leq k_{F}}\left\langle k k^{\prime}\left|G\left(\rho ; e(k)+e\left(k^{\prime}\right)\right)\right| k k^{\prime}\right\rangle_{a}
$$

The nuclear EoS can be calculated with good accuracy in the Brueckner two hole-line approximation with the continuous choice for the single-particle potential, since the results in this scheme are quite close to the calculations which include also the three hole-line contribution. 
However, as it is well known, the non-relativistic calculations, based on purely two- body interactions, fail to reproduce the correct saturation point of symmetric nuclear matter and one needs to introduce three- body forces (TBFs). In our approach the TBF's are reduced to a density dependent two-body force by averaging over the position of the third particle []. In this work we choose the Argonne $v_{18}$ nucleon-nucleon potential [ㅍ] , supplemented by the so-called Urbana model [1]8] as three-body force. This allows to reproduce correctly the nuclear matter saturation point $\rho_{0} \approx 0.16 \mathrm{fm}^{-3}, E / A \approx-16 \mathrm{MeV}$, and gives values of incompressibility and symmetry energy at saturation compatible with those extracted from phenomenology. For completeness we will show results obtained with the relativistic counterpart, i.e. the Dirac-Brueckner-Hartree-Fock scheme [ए9] where the Bonn A potential is used as NN interaction. In the low density region $\left(\rho<0.3 \mathrm{fm}^{-3}\right)$, both BHF+TBF binding energies and DBHF calculations are very similar, whereas at higher densities the DBHF is slightly stiffer [四]. This discrepancy can be understood by noticing that the DBHF treatment is equivalent to introducing in the nonrelativistic BHF the three-body force corresponding to the excitation of a nucleon-antinucleon pair, the so-called Z-diagram, which is repulsive at all densities.

The BBG approach has been extended to the hyperonic sector in a fully self-consistent way [미, [2]], by including the $\Sigma^{-}$and $\Lambda$ hyperons, but in this paper we mainly consider stellar matter as composed by neutrons, protons, and leptons in beta equilibrium, and only at the end we shall briefly discuss an example that includes the hyperonic degrees of freedom.

The chemical potentials of each species are the fundamental input for solving the equations of chemical equilibrium, charge neutrality and baryon number conservation, yielding the equilibrium fractions of all species. Once the composition of the $\beta$-stable, charge neutral stellar matter is known, one can calculate the equation of state, i.e., the relation between pressure $P$ and energy density $\varepsilon$ as a function of the baryon density $\rho$. It can be easily obtained from the thermodynamical relation

$$
\begin{aligned}
P & =-\frac{d E}{d V}=P_{B}+P_{l} \\
P_{B} & =\rho^{2} \frac{d\left(\varepsilon_{B} / \rho\right)}{d \rho}, \quad P_{l}=\rho^{2} \frac{d\left(\varepsilon_{l} / \rho\right)}{d \rho}
\end{aligned}
$$

with $E$ the total energy and $V$ the total volume. The total nucleonic energy density $\varepsilon_{B}$ is obtained by adding the energy densities of each species $\varepsilon_{i}$. As far as leptons are concerned, at those high densities electrons are a free ultrarelativistic gas, whereas muons are relativistic. Hence their energy densities $\varepsilon_{l}$ are well known from textbooks [22]. The numerical procedure has been often illustrated in papers and textbooks, and therefore it will not be repeated here.

\section{Quark Matter EoS : the Field Correlator Method}

The approach based on the FCM provides a natural treatment of the dynamics of confinement in terms of the Color Electric $\left(D^{E}\right.$ and $\left.D_{1}^{E}\right)$ and Color Magnetic $\left(D^{H}\right.$ and $\left.D_{1}^{H}\right)$ Gaussian correlators, being the former one directly related to confinement, so that its vanishing above the critical temperature implies deconfinement [ए]]. The extension of the FCM to finite temperature $T$ and chemical potential $\mu_{q}=0$ gives analytical results in reasonable agreement with lattice data thus 
allowing to describe correctly the deconfinement phase transition [23], 24]]. In this work, we are interested in the physics of neutron stars, and therefore the extension of the FCM to finite values of the chemical potential [23] allows to obtain the Equation of State of the quark-gluon matter in the range of baryon density typical of the neutron star interiors.

Within the FCM, the quark pressure for a single flavour is simply given by [23]

$$
P_{q} / T^{4}=\frac{1}{\pi^{2}}\left[\phi_{v}\left(\frac{\mu_{q}-V_{1} / 2}{T}\right)+\phi_{v}\left(-\frac{\mu_{q}+V_{1} / 2}{T}\right)\right]
$$

where

$$
\phi_{v}(a)=\int_{0}^{\infty} d u \frac{u^{4}}{\sqrt{u^{2}+v^{2}}} \frac{1}{\left(\exp \left[\sqrt{u^{2}+v^{2}}-a\right]+1\right)} .
$$

being $v=m_{q} / T$, and $V_{1}$, which is the result of the integration of a fundamental QCD correlator

$$
V_{1}=\int_{0}^{1 / T} d \tau(1-\tau T) \int_{0}^{\infty} d \chi \chi D_{1}^{E}\left(\sqrt{\chi^{2}+\tau^{2}}\right)
$$

indicates the large distance limit of the static $q \bar{q}$ potential : $V_{1} \equiv \lim _{x \rightarrow \infty} V_{1}(x)$. Therefore its value is an effective measure of the strength of the interaction when the particles are infinitely separated. Incidentally, this means that a change of sign of $V_{1}$ does not imply a change in the attractive or repulsive nature of the interaction which is instead related to the sign of the derivative of $V_{1}(x)$ and, on the same footing, $V_{1}=0$ does not exclude a finite interaction at short distance. The potential $V_{1}$ in Eq.(B.3) is assumed to be independent on the chemical potential, and this is partially supported by lattice simulations at very small chemical potential, as will be discussed below.

The EoS is completely specified once the gluon contribution is added to the quark pressure, i.e.

$$
P_{g} / T^{4}=\frac{8}{3 \pi^{2}} \int_{0}^{\infty} d \chi \chi^{3} \frac{1}{\exp \left(\chi+\frac{9 V_{1}}{8 T}\right)-1}
$$

and therefore

$$
P_{q g}=P_{g}+\sum_{j=u, d, s} P_{q}^{j}+\Delta \varepsilon_{v a c}
$$

where $P_{g}$ and $P_{q}^{j}$ are respectively given in Eq. (B.D) and (B.4), and

$$
\Delta \varepsilon_{v a c} \approx-\frac{\left(11-\frac{2}{3} N_{f}\right)}{32} \frac{G_{2}}{2}
$$

corresponds to the difference of the vacuum energy density between deconfined and confined phase, being $N_{f}$ the flavour number ( $N_{f}=3$ in this paper). $G_{2}$ is the gluon condensate whose numerical value, determined by the QCD sum rules, is known with large uncertainty [25]

$$
G_{2}=0.012 \pm 0.006 \mathrm{GeV}^{4}
$$

Therefore the EoS in Eq.(B.5) essentially depends on two parameters, namely the quark-antiquark potential $V_{1}$ and the gluon condensate $G_{2}$. It is interesting to notice that $G_{2}$ appears only in the vacuum contribution to the pressure (B.G), and plays the same role of the bag constant in the MIT bag model. In addition, if one turns $V_{1}$ off, $P_{q}$ becomes the pressure of free quarks, and in this case the FCM reduces to the simplest version of the bag model. Therefore $V_{1}$ can be regarded as 
the main correction to the free quarks dynamics inside the bag. Then, as the effect of confinement in the MIT bag model is accounted for by the presence of the bag constant, correspondingly in the FCM it is signaled by a negative vacuum pressure, and the transition to the deconfined phase corresponds to a change in the value of the bag or of the vacuum pressure.

To describe the deconfinement transition, we want to compare the hadronic pressure that by definition corresponds to the confined phase, and the quark matter pressure of the deconfined phase. To this purpose we only have to use the value of the vacuum pressure of the latter phase that is given in Eq.([3.6), as estimated in [[23], [24]. This is the main ingredient that governs the transition, while the inter-particle interaction $V_{1}$ provides a smaller correction to the deconfinement picture.

At finite temperature and vanishing baryon density, a comparison with the available lattice calculations of the Wuppertal-Budapest [26], and hotQCD collaborations [Q], provides clear indications about the specific values of the two parameters of the FCM, and in particular their values at the critical temperature $T_{c}$. These estimates are related to the values of the parameters at $T=\mu_{B}=0$ which, in turn, can be used as an input to study the EoS at $T=0$ and finite $\mu_{B}>0$.

In ref.[27] the EoS at zero baryon density has been derived, by explicitly assuming a temperature dependence of the gluon condensate $G_{2}$ as found in lattice simulations [28, 20], namely an almost constant $G_{2}(T)$ for $0<T<T_{c}$, with a sudden drop around $T_{c}$ to one half of its value, followed by the constant behavior $G_{2}(T)=G_{2}(T=0) / 2$, for $T>T_{c}$. In addition, an indication on the value of $V_{1}\left(T_{c}\right)$ has been extracted in [B]], starting from the expression of the critical temperature obtained in [2], []]

$$
T_{c}=\frac{a_{0} G_{2}^{1 / 4}}{2}\left(1+\sqrt{1+\frac{V_{1}\left(T_{c}\right)}{2 a_{0} G_{2}^{1 / 4}}}\right),
$$

where $a_{0}=\left(3 \pi^{2} / 768\right)^{1 / 4}$. In fact, once the values of $G_{2}$ and $T_{c}$ are fixed, one immediately gets $V_{1}\left(T_{c}\right)$ from Eq. (B.8), and in ref. [BO] it has been shown that, for $G_{2}(T=0)=0.012 \mathrm{GeV}^{4}$, the critical temperatures found in [32], [33], respectively $T_{c}=147 \pm 5 \mathrm{MeV}$ and $T_{c}=154 \pm 9 \mathrm{MeV}$, correspond to rather small values of $V_{1}\left(V_{1}\left(T_{c}\right) \lesssim 0.15 \mathrm{GeV}\right)$, while the optimum value indicated in [B]], $V_{1}\left(T_{c}\right)=0.5 \mathrm{GeV}$, reproduces those temperatures for small values of $G_{2}$, i.e. $G_{2} \simeq 0.004 \mathrm{GeV}^{4}$. However, one should recall that Eq.(B.8) is not extremely accurate, being obtained by neglecting the hadron pressure at the transition, which in [B] $]$ is estimated as a $10 \%$ uncertainty. Hence a check of the EoS focused on the critical point $T=T_{c}$ only, could be too restrictive, as the numerical data on lattice cover a large temperature range above $T_{c}$.

For that, we compare in Fig.W the predictions of the FCM with the available lattice data around and above the critical temperature. In Fig.W we concentrate on the interaction measure $(\varepsilon-3 p) / T^{4}$, which is particularly significant because it depends both on the energy density and on the pressure of the system and shows, around the critical temperature, large deviations from zero, i.e. the value of the interaction measure of a free gas of massless particles. The predictions of the FCM are checked against the lattice data for different parametrizations of $V_{1}(T)$, and also for constant $\mathrm{V}_{1}=0.01 \mathrm{GeV}$ and $\mathrm{V}_{1}=0.1 \mathrm{GeV}$. From Fig. $\mathrm{W}$ it is evident that these two constant values are too small, and higher values of $V_{1}$ must be considered. As suggested in ref.[B]], we take

$$
V_{1}(T)=c_{0}+V_{1}\left(\frac{T}{T_{c}}\right)=c_{0}+0.175\left(1.35 \frac{T}{T_{c}}-1\right)^{-1} \mathrm{GeV}
$$




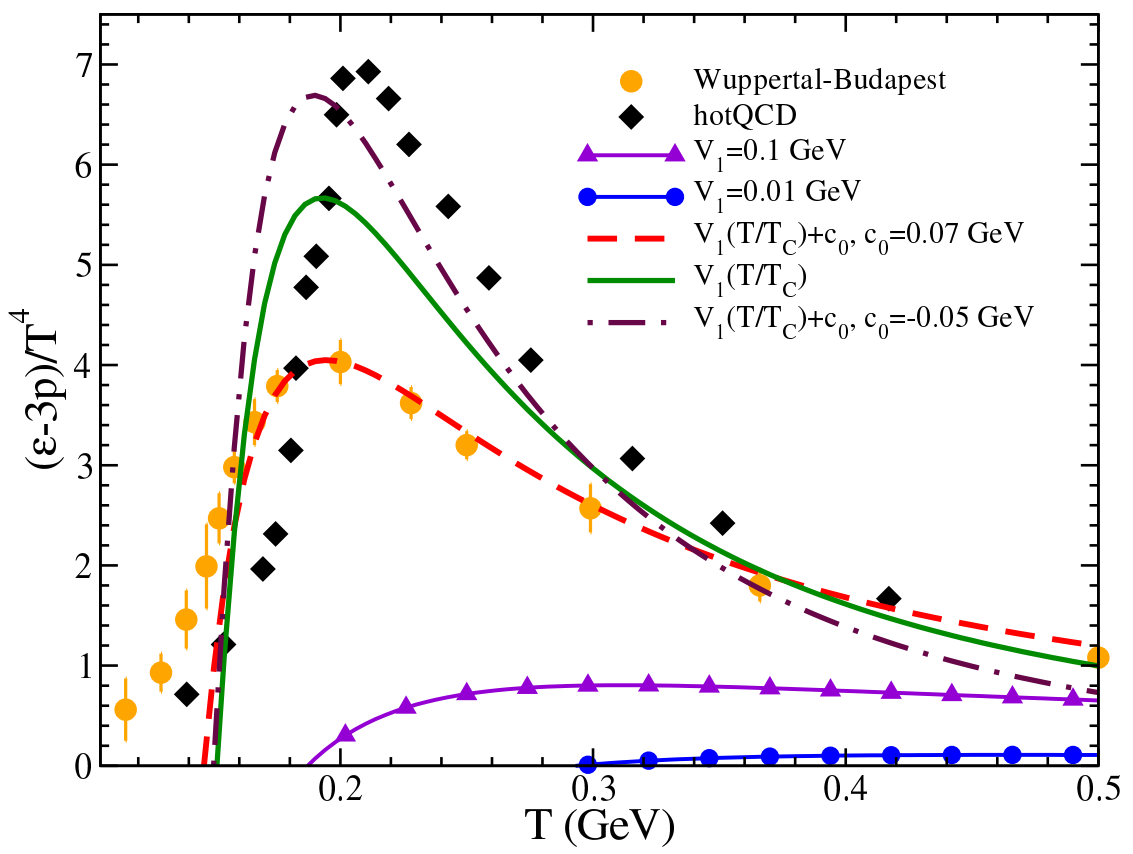

Figure 1: The interaction measure $(\varepsilon-3 \mathrm{p}) / \mathrm{T}^{4}$ as a function of the temperature as obtained in the FCM for three values of $c_{0}$ in Eq.(3.9) $: c_{0}=0.07,0,-0.05 \mathrm{GeV}$ (respectively: dashed (red), solid (green) and dotdashed (brown) curve), compared with the lattice data of ref.[26] (orange circles) and [9] (black, diamonds).

with respectively $\mathrm{c}_{0}=0.07,0,-0.05 \mathrm{GeV}$ (corresponding to $\mathrm{V}_{1}\left(\mathrm{~T}_{\mathrm{c}}\right)=0.57,0.5,0.45 \mathrm{GeV}$ ). The results, displayed by the dashed (red), solid (green) and dot-dashed (brown) curves, show a much better agreement with the data, and in particular the dashed (red) curve with $\mathrm{V}_{1}\left(\mathrm{~T}_{\mathrm{c}}\right)=0.57 \mathrm{GeV}$ gives a good fit to the data of [26], represented by full circles (orange), whereas $V_{1}\left(T_{c}\right)=0.45$ is preferable for the data in [ $[$ ] from the hotQCD collaboration, and shown as full diamonds (black). However, we warn that, more recently, the hotQCD collaboration is converging toward a smaller peak for $(\varepsilon-3 P) / T^{4}$ close to the Wuppertal-Budapest one. It is important to notice that there is no direct relation of these values with the potential at finite $\mu_{B}$. One would expect that an increasing baryon density could produce a screening effect that reduces the intensity of the quark-antiquark potential, and at large density the quark-quark interaction should become more and more relevant. Given those results, in our analysis we choose to keep $V_{1}$ as a free parameter, and check what kind of indications on $V_{1}$ can be extracted from the determination of the maximum mass of neutron stars.

Let us now turn to the other parameter of the FCM model, namely the gluon condensate $G_{2}$. It is interesting to notice that $G_{2}$ appears only in the vacuum contribution to the pressure, and it has the same role of the bag constant of the MIT bag model. As mentioned above, $G_{2}(T)$ at zero baryon density has been computed on lattice [28, 29] but, due to technical difficulties, analogous calculations in full QCD at large $\mu_{B}$ are precluded. In any case, because of the large uncertainty both on the value of this parameter and on its dependence on the chemical potential, we prefer to treat $G_{2}$ as the second free parameter of the FCM, as already done for $V_{1}$.

Typical effects that could induce changes with respect to the FCM picture are due to the colour superconductivity pairing mechanism, which is expected to take place for extremely large values of the chemical potential. These effects can be easily included in our analysis [B]]. In particular 
the possible additional contribution due to the new CFL phase, associated to quark-quark pairing, can be taken into account by adding the CFL pressure contribution to the full FCM pressure $P_{q g}$. The presence of color-flavour locked quark matter is expected at very high $\mu_{B}$, and it is realized through quark-quark pairing under the constraint that the densities of the three flavors, up, down and strange, are equal [35, [36, 37, 38]. The global effect of this pairing on the pressure is the presence of the additional term

$$
P_{c f l}=\frac{\Delta^{2} \mu_{B}^{2}}{3 \pi^{2}}
$$

only when the chemical potential is greater than $\mu_{B}=3 m_{s}^{2} /(4 \Delta)$ because, as discussed for instance in [38], a direct comparison of the grand potential with or without the inclusion of the CFL mechanism, indicates that this point marks the onset of pairing and consequently the appearance of the additional pressure term given in Eq.(B.TO), regardless of the particular values of $V_{1}$ and $G_{2}$ which parametrize the pressure of the FCM.

The gap $\Delta$ is expected to be in the range $10-100 \mathrm{MeV}$ in the region of interest of $\mu_{B}$ for the NS. Finally the total pressure of the quark matter phase is obtained by adding $P_{c f l}$ to $P_{q g}$ given in Eq. (B.5), and is treated as a function of the baryon chemical potential $\mu_{B}$ with three free parameters, namely the potential $V_{1}$ and the gluon condensate $G_{2}$, coming from the FCM model, and the gap $\Delta$, due to the CFL pairing.

\section{The hadron-quark phase transition}

Recently, it has been shown in ref.[II] that if a sharp phase transition occurs to a high-density phase, where the speed of sound is density-independent, then one observes the following four topologies of the mass-radius curve for compact stars as general feature of the nuclear to quark matter phase transition: the hybrid branch may be connected to the nuclear branch $(\mathrm{C})$, or disconnected (D), or both may be present (B) or neither (A). We use the term "hybrid branch" to refer to the part of the mass-radius relation of hybrid stars whose central pressure is above $p_{\text {trans }}$, and so they contain a core of the high-density phase. The occurrence of these as a function of the CSS parameters $p_{\text {trans }} / \varepsilon_{\text {trans }}$ and $\Delta \varepsilon / \varepsilon_{\text {trans }}$ at fixed $c_{\mathrm{QM}}^{2}$ is shown schematically in Fig. $\square$ (taken from Ref. [[4]]). The mass-radius curve in each region is depicted in inset plots, in which the thick green line is the hadronic branch, the thin solid red lines are stable hybrid stars, and the thin dashed red lines are unstable hybrid stars.

In the phase diagram the solid red line shows the threshold value $\varepsilon_{\text {crit }}$ below which there is always a stable hybrid star branch connected to the neutron star branch. This critical value is given by [3प, 40, 10]]

$$
\frac{\Delta \varepsilon_{\text {crit }}}{\varepsilon_{\text {trans }}}=\frac{1}{2}+\frac{3}{2} \frac{p_{\text {trans }}}{\varepsilon_{\text {trans }}}
$$

and was obtained by performing an expansion in powers of the size of the core of high-density phase. Eq.(ل) is an analytic result, independent of $c_{\mathrm{QM}}^{2}$ and the nuclear matter EoS. The dashed and dot-dashed black lines mark the appearance-disappearance of the connected or disconnected hybrid star branch. The position of these lines depends on the value of $c_{\mathrm{QM}}^{2}$ and (weakly) on the accompanying nuclear matter EoS [14]]. 


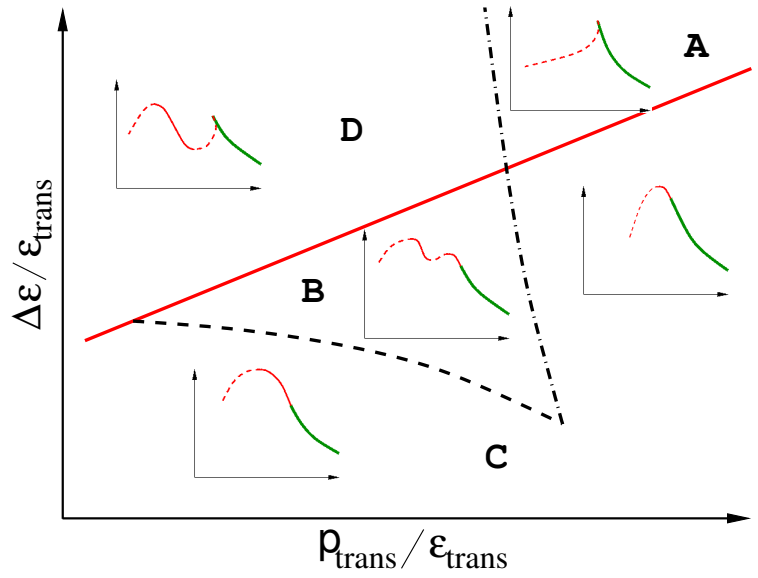

Figure 2: Schematic phase diagram (from [ए4]]) for hybrid star branches in the mass-radius relation of

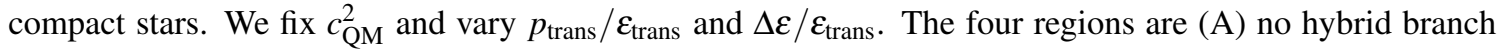
("absent"); (B) both connected and disconnected hybrid branches; (C) connected hybrid branch only; (D) disconnected hybrid branch only.
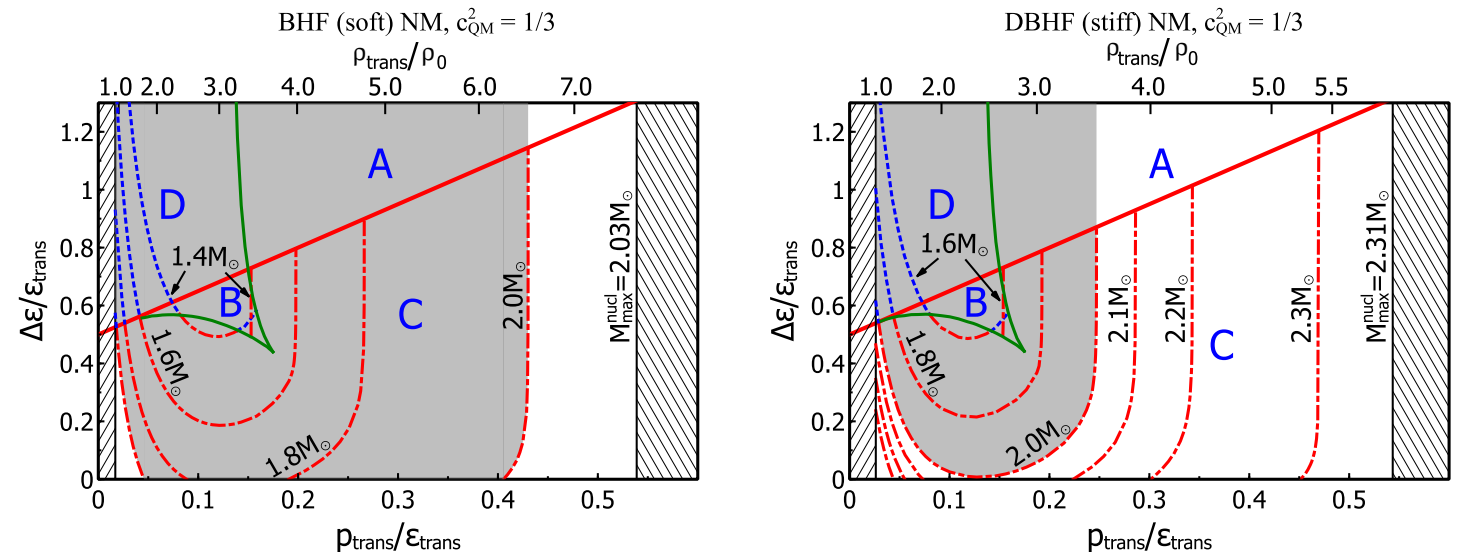

Figure 3: Contour plots showing the maximum hybrid star mass as a function of the CSS parameters of the high-density EoS. The left (right) plot is for a BHF (DBHF) nuclear matter EoS. The grey shaded region is excluded by the measurement of a $2 \mathrm{M}_{\odot}$ star. See text for details.

Once a nuclear matter EoS has been chosen, any high-density EoS that is well-approximated by the CSS parametrization can be summarized by giving the values of the three CSS parameters, corresponding to a point in the phase diagram. We then know what sort of hybrid branches will be present.

In Fig. B] we show how mass measurements of neutron stars can be expressed as constraints on the CSS parameters. Each panel shows the dependence on $p_{\text {trans }} / \varepsilon_{\text {trans }}$ and $\Delta \varepsilon / \varepsilon_{\text {trans }}$ for fixed $c_{\mathrm{QM}}^{2}$. The region in which the transition to quark matter would occur below nuclear saturation density $\left(\rho_{\text {trans }}<\rho_{0}\right)$ is excluded (hatched band at left end) because in that region bulk nuclear matter would be metastable. There is also a hatched band at the right hand of the figure indicating an upper limit on the transition pressure, which is the central pressure of the heaviest stable nuclear matter star. 
This depends on the hadronic EoS that had been assumed. The contours show the maximum mass of a hybrid star as a function of the EoS parameters. In particular the dot-dashed (red) contours are for hybrid stars on a connected branch, while the dashed (blue) are for disconnected branches. The region inside the $M=2 \mathrm{M}_{\odot}$ contour corresponds to EoS for which the maximum mass is less than $2 \mathrm{M}_{\odot}$ so it is shaded to signify that this region of parameter space for the high-density EoS is excluded by the observation of a star with mass $2 \mathrm{M}_{\odot}$ [ए]]. For high-density EoS with $c_{\mathrm{QM}}^{2}=1$ (not shown here), this region is extended over a large range of transition pressures and energy density discontinuities that are compatible with the observation. However, for high-density matter with $c_{\mathrm{QM}}^{2}=1 / 3$, which is the typical value in many models, the $M_{\max }>2 \mathrm{M}_{\odot}$ constraint eliminates a large region of the CSS parameter space [144, 42], leaving only a small white unshaded acceptable region. The left plot in Fig. B is for a softer nuclear matter EoS, Brueckner-Hartree-Fock (BHF) [U], whereas the right plot is for a stiffer nuclear matter EoS, Dirac-Brueckner-Hartree-Fock (DBHF)[प्] . As expected, the stiffer EoS gives rise to heavier (and larger) stars, and therefore allows a wider range of CSS parameters to be compatible with the $2 \mathrm{M}_{\odot}$ measurement.

From the left panel of Fig. B one can see that if, as predicted by many models, $c_{\mathrm{QM}}^{2} \lesssim 1 / 3$, then we are limited to two regions of parameter space, corresponding to a low pressure transition or a high pressure transition. In the low transition pressure region the transition occurs at a fairly low density $n_{\text {trans }} \lesssim 2 n_{0}$, and a connected hybrid branch is possible. In the high transition pressure region the connected branch (red dot-dashed) contours are, except at very low $\Delta \varepsilon$, almost vertical, corresponding to EoS that give rise to a very small connected hybrid branch which exists in a very small range of central pressures $p_{\text {cent }}$ just above $p_{\text {trans }}$. The maximum mass on this branch is therefore very close to the mass of the purely-hadronic matter star with $p_{\text {cent }}=p_{\text {trans }}$. The mass of such a purely hadronic star is naturally independent of parameters that only affect the quark matter EoS, such as $\Delta \varepsilon$ and $c_{\mathrm{QM}}^{2}$, so the contour is vertical.

Disconnected hybrid branches are of special interest, because they give a characteristic signature in mass-radius measurements. For both the hadronic EoS that we study, the regios B and D, where disconnected hybrid star branches can occur, are excluded for $c_{\mathrm{QM}}^{2} \leqslant 1 / 3$. Even for larger $c_{\mathrm{QM}}^{2}$ disconnected branches only arise if the nuclear matter EoS is sufficiently stiff. It is interesting to note that using an extremely stiff hadronic matter EoS such as DD2-EV [43] can further shrink the region that is excluded by the $M_{\max }>2 \mathrm{M}_{\odot}$ constraint, allowing disconnected branches of hybrid stars to occur.

\section{Mapping the FCM EoS on the CSS parametrization}

The CSS parametrization will be applicable to the FCM EoS if the speed of sound in the FCM EoS depends only weakly on the density or pressure. In Fig. 团 we show that this is indeed the case. The upper panel shows the speed of sound vs. pressure for different values of the FCM parameters, displayed in the lower panel. We see that the speed of sound varies by less than $5 \%$ over the considered range of pressures along each curve, and lies in the interval $0.28<c_{\mathrm{QM}}^{2}<1 / 3$. The value of $c_{\mathrm{QM}}^{2}$ shows a weak dependence on $V_{1}$ and extremely weak on $G_{2}$, which appears as an additive constant in the quark matter EoS according to Eq. (3.5). The transition pressure is more sensitive to the FCM parameters, increasing rapidly with $V_{1}$ and with $G_{2}$. The energy density at a given pressure increases slightly with $V_{1}$ or $G_{2}$. 


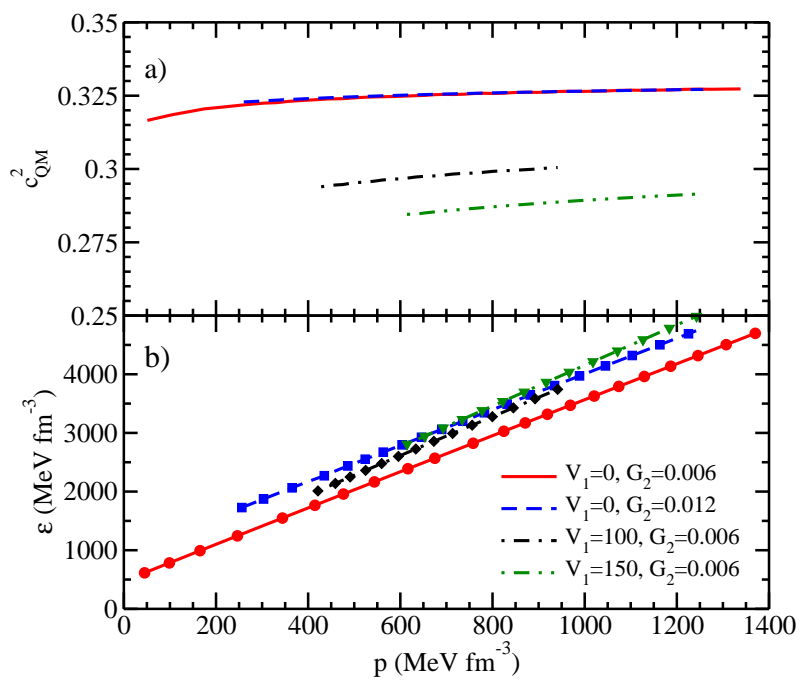

Figure 4: The squared speed of sound $c_{\mathrm{QM}}^{2}$ (panel (a)) is displayed vs. quark matter pressure for several values of $V_{1}$ (in $\mathrm{MeV}$ ) and $G_{2}\left(\right.$ in $\mathrm{GeV}^{4}$ ). In panel (b), the FCM energy density is represented by full symbols, whereas the full lines denote the CSS parametrization given by Eq.(1.1).

Let us now discuss the main features of the hadron-quark phase transition. Fig. $\square$ shows numerical results for the pressure as a function of the baryon chemical potential $\mu_{B}$ in the hadronic matter and quark matter in beta equilibrium. In particular, the green (red) solid curves represent the BHF (DBHF) EoS, whereas the remaining curves are the results for the FCM model with different choices of the quark-antiquark potential $V_{1}$ (expressed in $\mathrm{MeV}$ ). For completeness, a negative value of the potential, $V_{1}=-50 \mathrm{MeV}$ is also included in this analysis. In the left, middle and right panels the value assumed for the gap $\Delta$ is respectively equal to 0,50 and $100 \mathrm{MeV}$. All calculations shown in Fig. [ 5 are performed taking $G_{2}=0.006 \mathrm{GeV}^{4}$. We notice that with increasing the value of $V_{1}$ the transition point is shifted to larger values of the chemical potential, hence of the baryon density. However, the exact value depends also on the stiffness of the hadronic EoS at those densities. In this case, being the DBHF EoS stiffer than the BHF, the transition takes place at smaller values of the density. We notice that the transition point is affected also by the value of the gap $\Delta$, and is shifted toward smaller $\mu_{B}$ for larger value of the gap. We also see that no phase transition occurs for negative values of $V_{1}$.

The resulting EoS, for the several cases discussed, is the main input for solving the wellknown Tolman-Oppenheimer-Volkoff equations [22] for spherically symmetric NS, thus obtaining the mass-radius-central density relation. The mass of the NS has a maximum value as a function of radius (or central density), above which the star is unstable against collapse to a black hole. The value of the maximum mass depends on the EoS, so that the observation of a mass higher than the maximum mass allowed by a given EoS simply rules out that EoS. This is illustrated in Fig. 6 , where the relation between mass and radius (left panel) and central density (right panel) in units of the saturation density $\rho_{0}$ is displayed. Results are plotted for different values of $V_{1}, G_{2}$ and $\Delta$ and the BHF EoS is used for hadronic matter. The largest value of the maximum mass in this example is observed for large values of $V_{1}=200 \mathrm{MeV}, \Delta=100$ and $G_{2}=0.01$, and it is compatible with the largest mass observed up to now, i.e. $(2.01 \pm 0.04) M_{\odot}$ in PSR J0348+0432 [ए]]. 


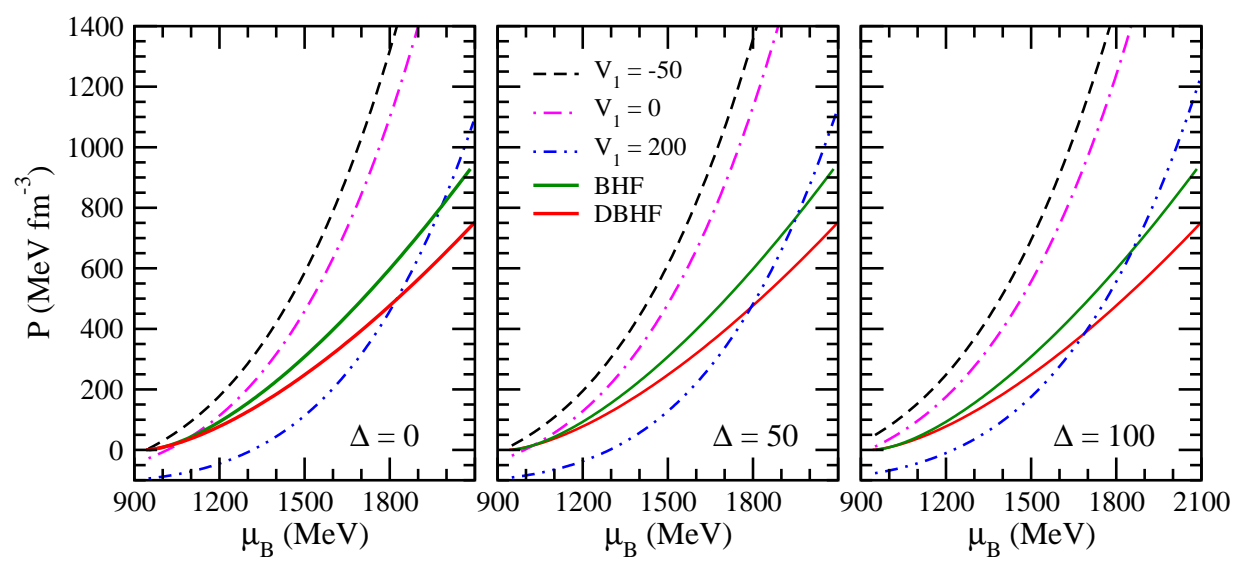

Figure 5: The pressure is displayed as a function of the baryon chemical potential $\mu_{B}$ for the FCM quark matter and the purely hadronic matter. All calculations for FCM have been performed for $G_{2}=0.006 \mathrm{GeV}^{4}$, and several values of $V_{1}$ have been chosen. The solid curves represent the BHF (green) and DBHF (red) EoS. Each panel shows results for different values of the gap $\Delta$, i.e. 0, 50, and $100 \mathrm{MeV}$.
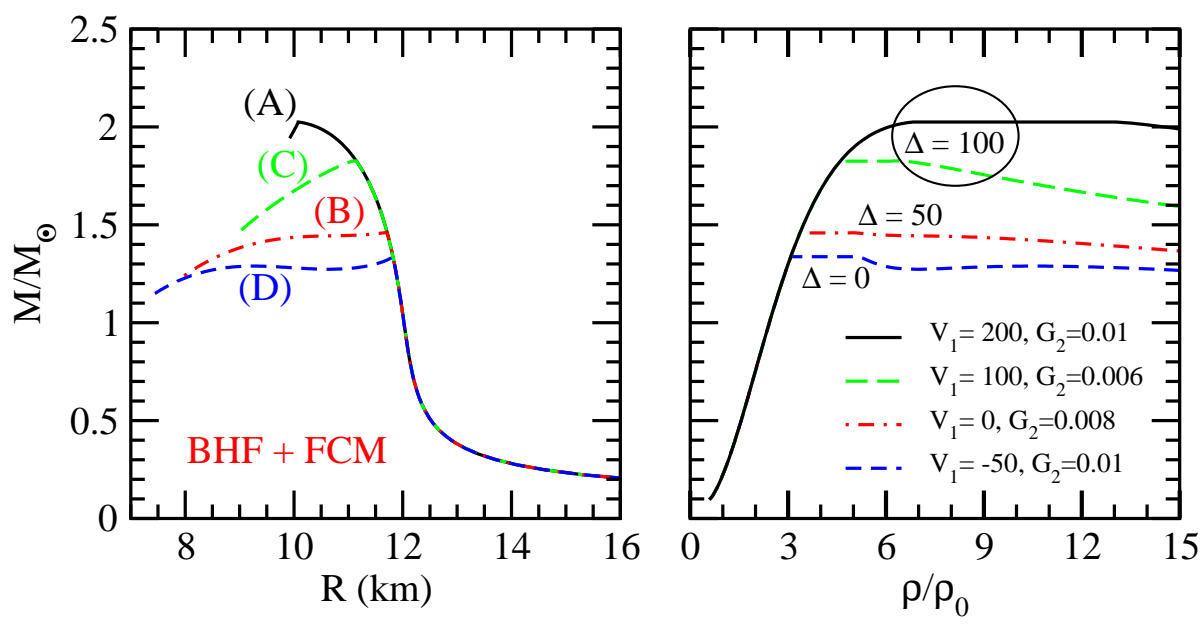

Figure 6: The mass as function of the radius (left panel) and the central density (right panel) is displayed for several values of $V_{1}, G_{2}$ and $\Delta$. The BHF EoS is used for the hadronic phase. The labels (A), (B), (C) and (D) indicate the specific topologies of the hybrid star branch.

In addition, in Figs. 6 we use the same labels as in Fig. $\square$ in order to indicate the topology of the mass-radius curve, which is strongly related to the chosen values of $V_{1}, G_{2}$ and $\Delta$. For example, when combining FCM quark matter to the BHF nuclear matter we find that, for unpaired quark matter and $V_{1}=-50 \mathrm{MeV}$, the lowest transition point can be obtained only if $G_{2}>0.006 \mathrm{GeV}^{4}$. Then, in Fig. 6 the mass-radius relation obtained with $G_{2}=0.01 \mathrm{GeV}^{4}$ is displayed by the blue dashed line and it exhibits a branch of stable hybrid stars disconnected (D) by the hadronic branch. With increasing $V_{1}$ the transition point moves to larger values of the pressure and the energy density, and as a consequence we explore regions of the phase diagram where the topology changes. For instance, for $V_{1}=0$ we can get both (B) connected and disconnected hybrid star branches, whereas 

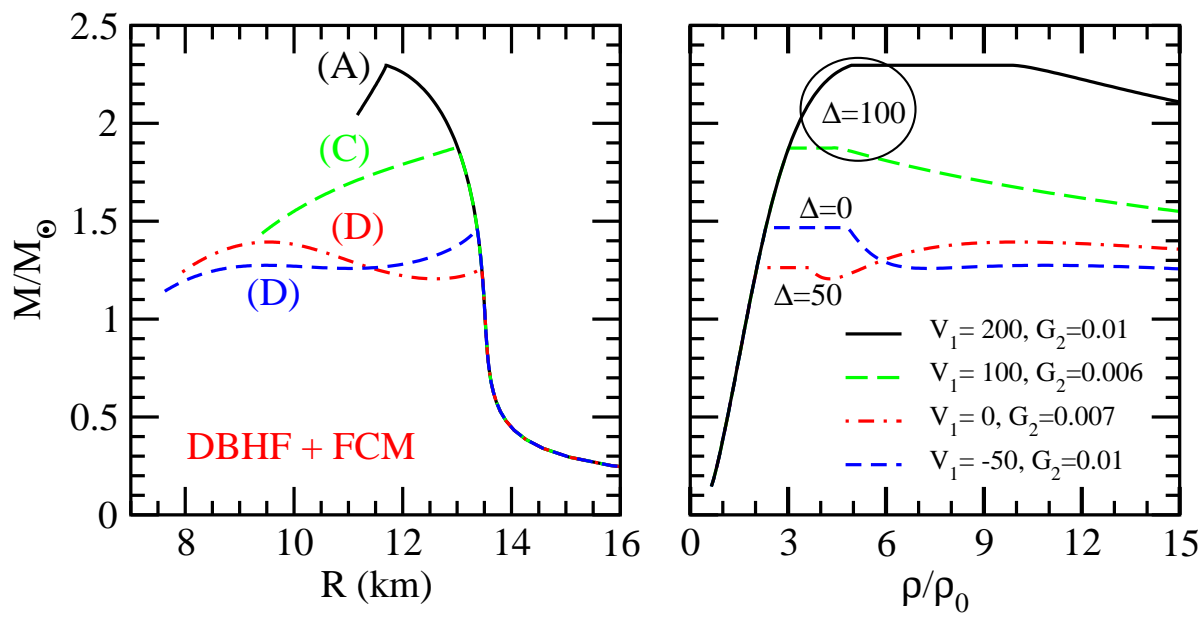

Figure 7: Same as Fig.6, but for the DBHF EoS.

for $V_{1}=100 \mathrm{MeV}$ connected (C) hybrid star branches are present and, for the largest value of $V_{1}=200 \mathrm{MeV}$ the hybrid branch is absent (A). This is clearly shown by a cusp in the mass-radius relation, and all configurations with radii smaller than the one characterizing the cusp are unstable. Therefore only purely nucleonic stars do exist in this case. However, the stability of those hybrid star configurations is related to the modeling of the deconfinement phase transition, as pointed out in ref.[4]4] where the Gibbs construction was used instead of the Maxwell method. The additional contribution of the CFL pressure to the FCM EoS produces only a shift of the transition point, and therefore the topology explored can be different than the one of the unpaired case. In Fig. $\square$ we display the mass-radius (left panel) and the mass-central density relation (right panel) when the DBHF EoS is used for the hadronic phase. We observe a topology similar to the one displayed in Fig. G, except for the (B) configurations, which do not appear for the chosen set of values used for $V_{1}, G_{2}$ and $\Delta$.

Let us now comment on the values of the maximum mass. In both cases, either BHF or DBHF EoS for the hadronic matter, we see that the largest possible values of the maximum mass are obtained only for values of $V_{1}>100 \mathrm{MeV}$, and that only in the DBHF case maximum masses well above the observational limit are possible. In fact, the heaviest BHF+FCM hybrid star has a mass of $2.03 M_{\odot}$, and the heaviest DBHF+FCM hybrid star has a mass of $2.31 M_{\odot}$. Those values are indicated by an orange cross in Fig. [8, where we display the mapping between the FCM and CSS parameters. In the upper (lower) panels we show results for the BHF (DBHF) hadronic EoS, whereas in the left, middle and right panels calculations are reported for different values of the gap $\Delta=0,50,100 \mathrm{MeV}$ respectively. The dashed black contour delimits the region accessible by the FCM calculation. Above that region, the symbols connected by solid lines show the CSS parametrization of the FCM quark matter EoS. Along each line we keep $V_{1}$ constant and vary $G_{2}$. In Fig. $8 V_{1}$ varies from $-50 \mathrm{MeV}$ up to the maximum value at which hybrid star configurations occur, which is indicated by an (orange) cross. For the BHF case that value is $V_{1}=240 \mathrm{MeV}$, $G_{2}=0.0024 \mathrm{GeV}^{4}$ and for the DBHF case it is $V_{1}=255 \mathrm{MeV}, G_{2}=0.0019 \mathrm{GeV}^{4}$. The vertical 
black dashed lines indicate the parameter regions accessible by the FCM and consistent with the measurement of a $M=2 M_{\odot}$. Hybrid stars with mass heavier than $2 M_{\odot}$ lie on a very small connected branch on the right side of the vertical black dashed lines, and cover a small range of central pressures, having a very tiny quark core, with mass and radius similar to those of the heaviest purely hadronic star, as was already discussed in Ref. [15]]. We have found that for a hybrid star with $\mathrm{M}=2 \mathrm{M}_{\odot}$ the radius of the quark core is bigger for the stiffest hadronic EoS, being comprised between 1 and $3 \mathrm{~km}$, whereas for the soft hadronic EoS the quark core radius is not larger that a few hundreds meters. In both cases the hadronic layer occupies the largest portion of the star, and is characterized by a radius of about $10 \mathrm{~km}$, whereas the crust radius is always smaller than $1 \mathrm{~km}$. Finally we briefly discuss the effects of adding the hyperon degrees of freedom in our
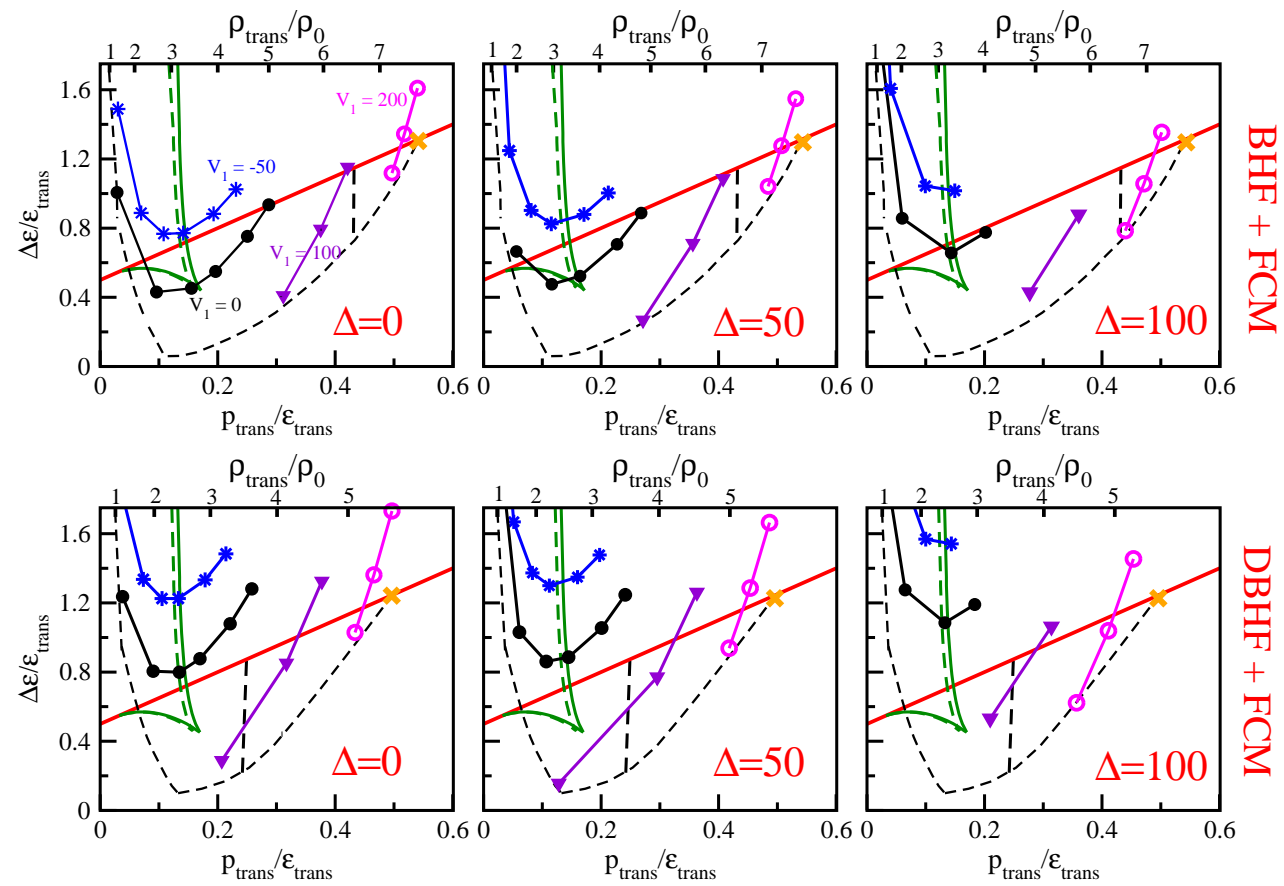

Figure 8: The mapping of the FCM quark matter model onto the CSS parametrization. Results are obtained using the BHF (upper panels) and DBHF (lower panels) nuclear matter EoS. The green curves are the phase boundaries for the occurrence of connected and disconnected hybrid branches. The dashed black line delimit the region yielded by the FCM model. Within that region, the symbols give CSS parameter values for FCM quark matter as $G_{2}$ is varied at constant $V_{1}$ (given in $\mathrm{MeV}$ ). The (orange) cross denotes the EoS with the highest $p_{\text {trans }}$, which gives the heaviest FCM hybrid star. The left, middle, and right panels display results obtained with $\Delta=0,50$, and $100 \mathrm{MeV}$ respectively.

analysis. In the past years, the BHF approach has been extended to include the hyperons $[41,42]$ and in fact, they are expected to appear in beta-stable matter already at relatively low densities of about twice nuclear saturation density which should produce a softening of the EoS with a strong decrease of the maximum mass. There exist several hyperon-nucleon (NY) potentials fitted to scattering data, i.e. NSC89 [43], NSC97 [44], and ESC08 [45], while the hyperon-hyperon (YY) potentials have presently to be considered rather uncertain or unknown, which is basically due to 
the lack of appropriate experimental data. Here we reconsider the phase transition to quark matter for the NSC89 and ESC08 potential and study the structure of the corresponding hybrid stars. As expected we observe that the softening of the hadronic EoS in the presence of hyperons is such that a double crossing of the hadronic and quark matter pressure can occur and, as a final effect, the maximum mass remains well below the $2 \mathrm{M}_{\odot}$ at variance with the observational constraint.

To bypass this obstacle we take the point of view of retaining only the first crossing of the hadronic and quark matter pressure curves and neglect the other crossing occurring at larger values of the chemical potential, which can be justified by our poor knowledge of the hyperon interactions. By following this procedure we report in Fig. $Q$ the mass-radius and mass-central density relation
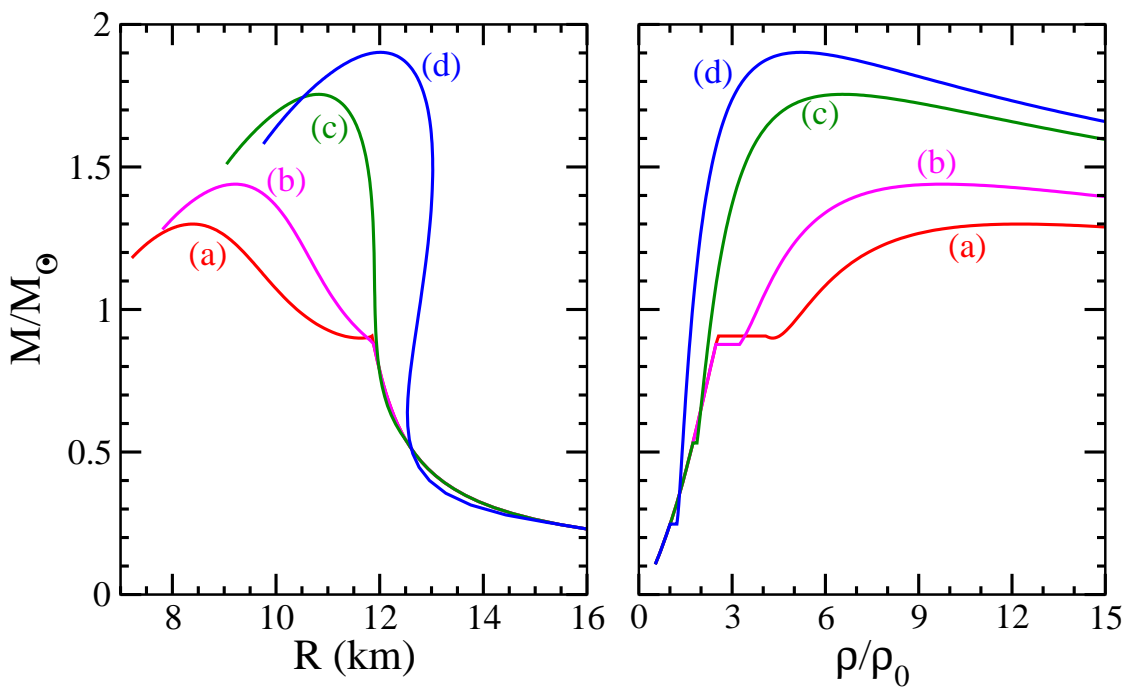

Figure 9: Mass-radius (left panel) and mass-central density (right panel) plots of the hybrid stars corresponding to the quark matter EoS (a), (b), (c), (d) together with the hyperon parametrization ESC08. See text for details.

of the hybrid stars corresponding to four different choices of the parameters of the FCM: $V_{1}=-50$ $\mathrm{MeV}$ (a), $V_{1}=0$ (b), $V_{1}=120 \mathrm{MeV}$ (c, d), with $\Delta=0$ in (a,b,d) and $\Delta=100 \mathrm{MeV}$ in (c). In addition, the two potentials NSC89 and ESC08 give qualitatively similar results and for convenience we display only the results obtained with the ESC08. It is evident that in the cases (a) and (b), when $V_{1}$ is small, the masses remain below $1.5 \mathrm{M}_{\odot}$ while in the other two cases with larger $V_{1}$, the mass of the NS grows above $1.7 M_{\odot}$ for (c) and up to $1.95 M_{\odot}$ for (d) which is reasonably close to the observational constraint of $2 M_{\odot}$. Only in this approach we are able to reach sufficiently high NS masses in the presence of hyperon degrees of freedom. It is remarkable that very similar results are obtained for the maximum NS masses in [45]] where the NSC89 parametrization is used for hyperons and a sort of QCD corrected bag model for quark matter. 


\section{Conclusions}

The FCM extension at finite $T$ and $\mu_{B}$ provides us a very simple description of the quark dynamics in terms of two parameters, namely the gluon condensate $G_{2}$, that parametrizes the vacuum pressure and energy density, and hence is strictly related to the bag constant of the MIT bag model, and the potential $V_{1}$, which summarizes the interaction corrections to the free quark and gluon pressure. In order to have a more complete picture, we include the effect of color superconductivity through the CFL mechanism, which amounts to the addition of a new free energy contribution written in terms of the gap $\Delta$.

Clearly, any prediction of a quark matter model on the structure of a hybrid NS strongly depends on the nuclear matter EoS employed, and among the large variety of nuclear EoS available in the literature, we focused on the non-relativistic BHF EoS and its relativistic counterpart, the DBHF EoS. We also analyzed the inclusion of the hyperon degrees of freedom that produces a softening of the nuclear matter EoS with the consequent reduction of the NS maximum mass.

With this new set of more refined calculations, we confirm the trend already observed in [13], 46, [5], i.e. the maximum mass of hybrid stars grows with the two parameters $V_{1}$ and $G_{2}$ while it decreases when $\Delta$ is increased. We also extend the mapping developed in [प5] among the parameters of the FCM and those defining the CSS parametrization, by displaying the effect of the gap $\Delta$, and we conclude that a particular configuration with mass around or above two solar masses can be realized in the FCM by different pairs of $G_{2}$ and $V_{1}$, depending on the specific value assigned to $\Delta$, i.e. the appearance of a color superconducting gap can be mimicked by a shift of the other two parameters. Therefore, even the mass of the heaviest hybrid star predicted by the FCM (the orange crosses in Fig. (5) does not correspond to a unique set of $G_{2}, V_{1}$ and $\Delta$, while, as seen in [ㄷ], its value strongly depends on the specific choice made for the nuclear matter EoS.

The inclusion of the hyperons induces dramatic changes in this picture. In fact, a regular transition from nuclear to quark matter with a stable quark phase up to very high chemical potential requires a particular tuning of the FCM parameters that leads to low maximum masses not compatible with the heaviest observed NS. At the same time, we noticed that this problem can be softened if one retains only the crossing from the hadronic to the quark matter phase at lowest $\mu_{B}$ and neglects the other transitions at higher chemical potential. Then, under this hypotesis it is possible to tune the FCM parameters in such a way to raise the maximum mass up to $1.95 M_{\odot}$.

\section{Acknowledgments}

Partial support comes from "NewCompStar", COST Action MP1304.

\section{References}

[1] G. Taranto, M. Baldo, and G. Burgio, Selecting microscopic Equations of State, Phys.Rev. C87 (2013) 045803, [arXiv: 1302.6882].

[2] M. Baldo, I. Bombaci, and G. Burgio, Microscopic nuclear equation of state with three-body forces and neutron star structure, Astron.Astrophys. 328 (1997) 274-282, [astro-ph/9707277].

[3] A. Akmal, V. R. Pandharipande, and D. G. Ravenhall, Equation of state of nucleon matter and neutron star structure, Phys.Rev. C58 (1998) 1804-1828, [nuc1-th/9804027]. 
[4] X. R. Zhou, G. F. Burgio, U. Lombardo, H.-J. Schulze, and W. Zuo, Three body forces and neutron star structure, Phys.Rev. C69 (2004) 018801.

[5] M. Baldo, A. Polls, A. Rios, H.-J. Schulze, and I. Vidaña, Comparative study of neutron and nuclear matter with simplified Argonne nucleon-nucleon potentials, Phys.Rev. C86 (2012) 064001, [arXiv:1207.6314]].

[6] Z. Li, U. Lombardo, H.-J. Schulze, and W. Zuo, Consistent nucleon-nucleon potentials and three-body forces, Phys.Rev. C77 (2008) 034316.

[7] P. de-Forcrand, Simulating QCD at finite density, in Symposium on Lattice Field Theory, p. 10, 2009.

[8] Y. Aoki, G. Endrődi, Z. Fodor, S. D. Katz, and K. K. Szabó, The order of the quantum chromodynamics transition predicted by the standard model of particle physics, Nature $\mathbf{4 4 3}$ (2006) 675-678, [hep-1at/0611014].

[9] A. Bazavov, T. Bhattacharya, M. Cheng, N. H. Christ, C. Detar, S. Ejiri, S. Gottlieb, R. Gupta, U. M. Heller, K. Huebner, C. Jung, F. Karsch, E. Laermann, L. Levkova, C. Miao, R. D. Mawhinney, P. Petreczky, C. Schmidt, R. A. Soltz, W. Soeldner, R. Sugar, D. Toussaint, and P. Vranas, Equation of state and QCD transition at finite temperature, Phys. Rev. D80 (2009) 014504, [arxiv:0903.4379].

[10] J. Antoniadis, P. Freire, N. Wex, T. Tauris, R. Lynch, and et al., A Massive Pulsar in a Compact Relativistic Binary, Science 340 (2013) 1233232, [arXiv:1304.6875].

[11] A. Di Giacomo, H. G. Dosch, V. Shevchenko, and Y. Simonov, Field correlators in QCD: Theory and applications, Phys.Rept. 372 (2002) 319-368, [hep-ph/0007223].

[12] M. Baldo, G. F. Burgio, P. Castorina, S. Plumari, and D. Zappala, Quark matter in neutron stars within the Nambu - Jona-Lasinio model and confinement, Phys. Rev. C75 (2007) 035804, [hep-ph/0607343].

[13] M. Baldo, G. Burgio, P. Castorina, S. Plumari, and D. Zappala, Astrophysical constraints on the confining models: The Field Correlator Method, Phys.Rev. D78 (2008) 063009, [arxiv:0804.2328].

[14] M. G. Alford, S. Han, and M. Prakash, Generic conditions for stable hybrid stars, Phys.Rev. D88 (2013) 083013, [arXiv:1302.4732].

[15] M. G. Alford, G. F. Burgio, S. Han, G. Taranto, and D. Zappalà, Constraining and applying a generic high-density equation of state, Phys. Rev. D92 (2015) 083002, [arXiv: 1501.0790].

[16] M. Baldo, ed., Nuclear Methods and The Nuclear Equation of State. World Scientific, Singapore, 1999.

[17] R. B. Wiringa, V. Stoks, and R. Schiavilla, An Accurate nucleon-nucleon potential with charge independence breaking, Phys.Rev. C51 (1995) 38-51, nuc1-th/9408016.

[18] J. Carlson, V. Pandharipande, and R. B. Wiringa, Three-nucleon interaction in 3-body, 4-body, and infinite-body systems, Nucl.Phys. A401 (1983) 59-85.

[19] T. Gross-Boelting, C. Fuchs, and A. Faessler, Covariant representations of the relativistic Bruckner T matrix and the nuclear matter problem, Nucl.Phys. A648 (1999) 105-137, [nuc1-th/9810071].

[20] M. Baldo, G. Burgio, and H. Schulze, Onset of hyperon formation in neutron star matter from Brueckner theory, Phys.Rev. C58 (1998) 3688-3695. 
[21] M. Baldo, G. Burgio, and H. Schulze, Hyperon stars in the Brueckner-Bethe-Goldstone theory, Phys.Rev. C61 (2000) 055801, nuc1-th/9912066].

[22] S. L. Shapiro and S. A. Teukolsky, Black Holes, White Dwarfs and Neutron Stars: The Physics of Compact Objects. 1986.

[23] Y. Simonov and M. Trusov, Deconfinement transition for nonzero baryon density in the field correlator method, JETP Lett. 85 (2007) 598-601, [hep-ph/0703228].

[24] Y. Simonov and M. Trusov, Vacuum phase transition at nonzero baryon density, Phys.Lett. B650 (2007) 36-40, [hep-ph/0703277].

[25] M. A. Shifman, A. Vainshtein, and V. I. Zakharov, QCD and Resonance Physics. Sum Rules, Nucl.Phys. B147 (1979) 385-447.

[26] S. Borsányi, G. Endrődi, Z. Fodor, A. Jakovác, S. D. Katz, S. Krieg, C. Ratti, and K. K. Szabó, The QCD equation of state with dynamical quarks, Journal of High Energy Physics 11 (2010) 77, [arxiv: 1007.2580$]$ ].

[27] Yu. A. Simonov and M. A. Trusov, Deconfinement transition for nonzero baryon density in the field correlator method, JETP Lett. 85 (2007) 598-601, [hep-ph/0703228].

[28] M. D'Elia, A. Di Giacomo, and E. Meggiolaro, Field strength correlators in full QCD, Phys. Lett. B408 (1997) 315-319, [hep-1at/9/05032].

[29] M. D'Elia, A. Di Giacomo, and E. Meggiolaro, Gauge invariant field strength correlators in pure Yang-Mills and full QCD at finite temperature, Phys. Rev. D67 (2003) 114504, [hep-1at/0205018].

[30] I. Bombaci and D. Logoteta, A link between measured neutron star masses and lattice QCD data, Monthly Notices of the Royal Astronomical Society - Letters, Vol. 433, L79-L83 (2013) [arxiv: 1212.5907].

[31] Yu. A. Simonov and M. A. Trusov, Vacuum phase transition at nonzero baryon density, Phys. Lett. B650 (2007) 36-40, [hep-ph/0703277].

[32] S. Borsanyi, Z. Fodor, C. Hoelbling, S. D. Katz, S. Krieg, C. Ratti, and K. K. Szabo, Is there still any Tc mystery in lattice QCD? Results with physical masses in the continuum limit III, JHEP 09 (2010) 073, [arXiv: 1005.3508].

[33] A. Bazavov et. al., The chiral and deconfinement aspects of the QCD transition, Phys. Rev. D85 (2012) 054503, [arXiv:11]1.1710].

[34] G. F. Burgio and D. Zappala', Hybrid star structure with the Field Correlator Method, ArXiv e-prints (Sept., 2015) [arXiv: 1509.0084].

[35] M. G. Alford, A. Schmitt, K. Rajagopal, and T. Schäfer, Color superconductivity in dense quark matter, Rev.Mod.Phys. 80 (2008) 1455-1515, [arXiv:0709.4635].

[36] K. Rajagopal and F. Wilczek, Enforced electrical neutrality of the color flavor locked phase, Phys.Rev.Lett. 86 (2001) 3492-3495, [hep-ph/0012039].

[37] M. Alford and K. Rajagopal, Absence of two flavor color superconductivity in compact stars, JHEP 0206 (2002) 031, [hep-ph/020400]].

[38] M. Alford, M. Braby, M. Paris, and S. Reddy, Hybrid stars that masquerade as neutron stars, Astrophys.J. 629 (2005) 969-978, nucl-th/0411016]. 
[39] Z. F. Seidov, The Stability of a Star with a Phase Change in General Relativity Theory, Sov. Astron. 15 (1971) 347.

[40] R. Schaeffer, L. Zdunik, and P. Haensel, Phase transitions in stellar cores. I - Equilibrium configurations, Astron.Astrophys. 126 (1983) 121-145.

[41] L. Lindblom, Phase transitions and the mass radius curves of relativistic stars, Phys.Rev. D58 (1998) 024008, [ar-qc/9802072].

[42] P. F. Bedaque and A. W. Steiner, Sound velocity bound and neutron stars, Phys.Rev.Lett. 114 (2015) 031103, [arXiv: 1408.5116].

[43] S. Benic, D. Blaschke, D. E. Alvarez-Castillo, T. Fischer, and S. Typel, A new quark-hadron hybrid equation of state for astrophysics - I. High-mass twin compact stars, arXiv:1411.2856.

[44] D. Logoteta and I. Bombaci, Quark deconfinement transition in neutron stars with the field correlator method, Phys. Rev. D88 (2013) 063001, [arXiv:1309.0096].

[45] A. Kurkela, P. Romatschke, and A. Vuorinen, Cold Quark Matter, Phys.Rev. D81 (2010) 105021, [arxiv:0912.1856].

[46] S. Plumari, G. Burgio, V. Greco, and D. Zappala, Quark matter in Neutron Stars within the Field Correlator Method, Phys.Rev. D88 (2013) 083005, [arXiv: 1307.3055]. 